\title{
Experimentos de orientación teórica. Una discusión metodológica ${ }^{1}$
}

\author{
Luis Miguel Miller Moya \\ nstituto de Estudios Sociales Avanzados de Andalucía (IESA-CSIC) \\ Immiller@iesaa.csic.es
}

Recibido: $10-06.2006$

Aceptado: 20.11 .2006

\section{INTRODUCCIÓN}

¿Tiene sentido la aplicación del método experimental en sociología? En un momento en el que se está produciendo una notable discusión metodológica en torno a la pertinencia de dicho método en otras ciencias sociales, como la economía y la ciencia política, no está de más que reflexionemos si el mismo tiene cabida en la sociología actual. Pero, como pondremos de manifiesto en el presente artículo, hablar de método experimental en general supone algo tan ambiguo como referirnos a los métodos no-experimentales o la metodología cualitativa en general. Es por ello que, en aras de la claridad de la exposición, nuestra discusión quedará circunscrita a un tipo particular de experimento.

El tipo de diseño experimental que va a ser objeto de análisis en este artículo se ajusta a lo que Ernest Greenwood denominaba «experimento puro ${ }^{2}$ », un tipo de diseño que supone el «ideal de método experimental, el modelo hacia el que todos los demás tipos deberían aspirar» (Greenwood, 1978: 44). Este trabajo está dedicado a una discusión metodológica en torno a dicho método, sus potencialidades, así como los problemas que entraña su aplicación. Sin embargo, antes de proceder a detenernos sobre el tipo de «experimento puro» en particular, nos parece pertinente una presentación del método experimental de forma ge-

${ }^{1}$ Agradezco la financiación recibida del Centro de Estudios Andaluces a través del proyecto SOCH2.05/43-2006. El texto se ha beneficiado también de los comentarios recibidos en las presentaciones realizadas en el Instituto de Estudios Sociales Avanzados (IESA-CSIC) en junio de 2006 y en el Grupo de Teoría y Metodología del III Congreso Andaluz de Sociología en noviembre de 2006.

2 Otras etiquetas, que pueden resultar más intuitivas, han sido empleadas para denominar este tipo de diseños experimentales, tales como: «método de laboratorio», «experimento directo», "experimento controlado», «experimento científico», «experimento verdadero» o «experimento de laboratorio», 
nérica que nos sirva para situar en un contexto más amplio el tipo de diseño que va a ser discutido.

El método experimental no ha ocupado, ni ocupa, un lugar privilegiado en el arsenal metodológico propio del sociólogo. Su aplicación en esta disciplina queda lejos no sólo de su uso en psicología - la ciencia humana experimental por excelencia - sino incluso lejos de su empleo actual en otras ciencias que estudian el comportamiento humano, como la economía o la ciencia política. Por tanto, no parece descabellado aprovechar la discusión metodológica de una mayor raigambre presente en estas otras disciplinas para defender el uso de dicho método en sociología. Sin embargo, aunque haremos uso de ella, nuestro propósito es anclar nuestra reflexión en el debate en torno al método experimental que se ha producido en el seno de la propia sociología.

Como veremos, la discusión sociológica a propósito de este método ha girado en torno a dos grandes temas. El primero hace referencia a la delimitación conceptual de aquello a lo que podemos denominar experimento, así como en qué medida el método experimental difiere de otros métodos empleados por la disciplina sociológica. El segundo tema apunta directamente al problema de la validez general de dicho método y a la pertinencia de su aplicación al estudio de fenómenos sociales. Ambos aspectos constituyen la columna vertebral de este trabajo.

Empezando por el primero de los temas planteados, y antes de proceder a proporcionar varias definiciones acerca de lo que aquí entendemos por método experimental, nos preguntamos: ¿qué características básicas se encuentran presentes en la mayor parte de las investigaciones que se definen a sí mismas como experimentales? De acuerdo con Greenwood $(1978: 29)^{3}$, que a su vez se apoya en la seminal obra de John Stuart Mill (1891), el método experimental queda definido por tres características básicas, a saber:

(1) la presencia de una hipótesis causal;

(2) que es estudiada a través de una serie de situaciones de contraste;

(3) estas situaciones de contraste han sido controladas.

${ }^{3}$ Puede sorprender el presente uso de una obra como la de Greenwood (1978) -cuya primera edición data de 1945 - para aproximamos a las características básicas del método experimental. De hecho, a lo largo del artículo se utilizan otra serie de referencias, tanto sociologicas como procedentes de otras ciencias sociales, bastante más actuales. Sin embargo, la obra de Greenwood cumple varios requisitos difíciles de encontrar en obras más recientes. En primer lugar, es una de las pocas monografías - quizá la única junto con la de Chapin (1947) — dedicadas íntegramente a una discusión metodológica de la aplicación del método experimental en sociología. En segundo lugar, como Oakley (1998) ha puesto de manifiesto, se trata de una de las referencias básicas utilizadas por los sociólogos que, durante las décadas de los sesenta y setenta del pasado siglo, utilizaron el método experimental como herramienta privilegiada para el análisis sociologico, sobre todo en lo que se refiere a programas de evaluación de toda índole. En tercer lugar, el libro está escrito en un momento en el que existía un amplio debate acerca del método experimental en sociología, como pone de manifiesto Paul Lazarsfeld en la introducción del propio libro. Por último, Greenwood no se compromete con la utilización de un diseño experimental u otro, sino que pretende evaluar las luces y sombras de cada tipo particular de diseño, lo que nos puede ser muy útil para situar el tipo de diseño experimental concreto en el que nos detendremos más adelante. 
Nuestro planteamiento de partida asume que para que podamos añadir el adjetivo experimental a una investigación, estas tres características deben estar en algún grado presentes. Este hecho, a pesar de acotar nuestra definición de lo que es un experimento, todavía permite imaginar diseños de investigación bastante diferentes atendiendo a cómo cada una de estas características sea puesta en práctica. Así, el componente de causalidad puede ser entendido en el sentido cronológico, como una relación temporal asimétrica, donde la causa precede al efecto o puede ser entendido como la regularidad en la presencia conjunta de dos hechos que no implica asimetría temporal alguna, como ocurre cuando hacemos referencia al carácter reflexivo del comportamiento en sociedad. Por otra parte, las situaciones de contraste pueden proceder de la observación de un fenómeno natural o pueden haber sido creadas por el propio investigador, como ocurre en el caso de los experimentos de laboratorio que describiremos a continuación. Por último, también el control ejercido sobre dichas situaciones de contraste puede ser llevado a cabo de muy diversas formas. Por ejemplo, el control puede ser ejercido sobre el desempeño del investigador, como en las situaciones en las que dos o más investigadores observan y codifican el mismo fenómeno independientemente para garantizar un mayor grado de objetividad sobre lo observado. Pero el control también puede ejercerse sobre el propio objeto de estudio, por ejemplo utilizando grupos de contraste que sean similares en todas las características imaginables menos una, que es la que constituye el objeto de investigación. Esta heterogeneidad de los diseños que podemos clasificar como experimentales nos llevará en el próximo apartado a contraponer varios tipos de diseños experimentales antes de adentrarnos en la descripción de un diseño en particular. Pero antes de esto, y a la luz de las características introducidas más arriba, proporcionaremos algunas definiciones específicas del método experimental.

A partir de una abstracción de las características particulares de cada tipo de diseño experimental, Greenwood (1978) define un experimento del siguiente modo:

Un experimento es la comprobación de una hipótesis que busca relacionar dos factores en una relación causal a través del estudio de situaciones de contraste en las que todos los factores, excepto aquel que interesa [estudiar], son controlados; este factor es bien la causa hipotética o bien el efecto hipotético (p. 28).

Como se observa, esta definición sólo supone una combinación de las tres características introducidas anteriormente. La misma todavía no excluye ninguno de los tipos de diseños experimentales que presentaremos en el apartado siguiente. Nótese que la definición no incluye ninguna referencia a que el experimento conlleve una manipulación de las variables por parte del investigador. Este elemento de manipulación, sin embargo, constituye la característica fundamental del método experimental para los investigadores sociales contemporáneos. Sirva como ejemplo la definición propuesta por Levin (1999): 
[El método experimental] es el método que el investigador elige cuando está principalmente interesado en determinar las causas y los efectos. El método experimental está caracterizado por la manipulación de una o más variables denominadas variables independientes y el control de todas las demás. Manipulación quiere decir crear o seleccionar niveles discretos de una variable y comparar las respuestas obtenidas en los distintos niveles. Control significa mantener una variable superflua (una variable distinta a la variable independiente que puede influenciar los resultados de un experimento) constante en los distintos niveles de la variable independiente. Si el investigador consigue controlar todas las variables superfluas, entonces él o ella puede determinar que los cambios en el comportamiento (la variable, o variables, dependiente) deben haber sido causados por la manipulación de la variable independiente (p. 5).

Incluso hay quien va más allá y afirma que es precisamente la manipulación de la variable independiente lo que separa el método experimental de los métodos no-experimentales en la ciencia social, como puede leerse en la siguiente cita correspondiente a la introducción que Michael Lewis-Beck realiza al trabajo de Brown y Melamed (1990):

Los métodos de la ciencia social pueden ser clasificados en dos [tipos], noexperimental y experimental. Un principio clave del trabajo experimental es la manipulación de la variable de tratamiento $(X)$, seguido de la observación de una variable de respuesta $(Y)$. (Brown y Melamed, 1990: v).

Estas citas ponen de relieve una primera diferencia entre lo que denominaremos como experimentos de orientación empírica y experimentos de orientación teórica. En los siguientes apartados profundizaremos en otros elementos adicionales que nos permiten establecer sendas diferencias entre ambas concepciones $^{4}$.

${ }^{4}$ No constituye un objetivo central de este trabajo indagar sobre las diferentes etapas en la aplicación del método experimental en sociología. A pesar de ello, por conveniencia, utilizaremos las etiquetas de experimentos de orientación empírica y experimentos de orientación teórica para referimos a dos momentos diferentes en el uso dado por los sociólogos a dicho método. Las características de ambos momentos serán desveladas a lo largo de este artículo, así que no abundaremos más en ellas en este momento. En cuanto a su localización temporal diremos que la orientación empírica cubriría un período de unos sesenta años, desde comienzos de los años veinte hasta finales de los años setenta. Dos obras claves en este extenso período son los ensayos metodologicos de Chapin (1947 [1931]) y de Greenwood (1978 [1945]). Según el relato de Oakley (1998), en la década de los ochenta se produce un descrédito de lo que hemos denominado experimentos de orientación empírica. La denominada orientación teórica es bastante más reciente y, a grandes rasgos, supone la traslación a la sociología de la «revolución del comportamiento» que ha tenido lugar en los últimos veinte años en disciplinas como la economía, en primer lugar, y la ciencia política, en segundo. Aunque ya encontramos trabajos a lo largo de los años ochenta y noventa, una fecha clave es, sin duda, la concesión del premio Nobel de economía al economista experimental Vemon Smith y al psicólogo Daniel Kahneman en el año 2002 por haber aplicado con éxito el método experimental al estudio de fenómenos económicos. 


\section{DOS ORIENTACIONES DISTINTAS DE LA EXPERIMENTACIÓN}

Resulta obvio afirmar que no todos los experimentos son iguales ni sirven para lo mismo. En la introducción de este artículo hemos señalado tres características comunes a todo experimento, pero a partir de ahí existen múltiples diseños posibles. En este apartado vamos a tratar de responder una pregunta básica: ¿para qué le sirve al sociólogo el método experimental? Algunos sociólogos dirían que el método experimental proporciona al investigador una forma de descubrir nuevos datos, así como las relaciones que se pueden establecer entre estos, presentes en la realidad social. En este caso, ante una situación social existente, la aplicación ex post del método de investigación permite al investigador reconstruir tales relaciones y en última instancia atribuirles causalidad, es decir, determinar las causas y los efectos correspondientes a la situación en cuestión. Otros podrían responder que el método experimental sirve para contrastar las hipótesis teóricas elaboradas ex ante por el científico social. En este caso, las hipótesis teóricas, independientemente de cuál sea su procedencia ${ }^{5}$, son sometidas a un estudio riguroso, preferentemente en un entorno que permita la manipulación explícita de las variables que se hallan en la base de dichas hipotesis. Denominaremos la primera de estas respuestas intuitivas orientación empírica del método experimental y la segunda orientación teórica. La diferencia entre ambas concepciones no es trivial y necesita ser aclarada.

\section{Experimentos de orientación empírica}

La experimentación de orientación empírica vivió su momento álgido entre las décadas de los sesenta y de los ochenta en Estados Unidos, aunque se pueden encontrar ejemplos con anterioridad a la Segunda Guerra Mundial ${ }^{6}$. La Tabla 1 muestra el abanico de temas, objetivos, diseños de investigación y resultados obtenidos por distintos sociólogos que utilizaron el método experimental a lo largo de estas décadas. Como se observa, este método fue utilizado para evaluar la eficacia de numerosos programas de intervención social; desde programas de ayuda a la incorporación al mercado de trabajo, hasta programas relacionados con la reinserción social de ex presidiarios. La metodología empleada por los mismos seguía siempre un patrón parecido, consistente en la comparación de diferentes grupos de individuos a los que, de forma aleatoria, se les sometía a algún tipo de intervención social. Así, por ejemplo, se comparaban los resultados obtenidos tras un período determinado de tiempo de ex presidiarios que habían participado en un programa de reinserción social con otros que no habían tenido dicha

${ }^{5}$ Estas hipótesis teóricas pueden proceder tanto de investigaciones empíricas precedentes, como de argumentaciones lógico-deductivas apoyadas en teorías cuyo alcance y condiciones de posibilidad estén bien definidos, como es el caso de la teoría de la elección racional.

6 Ver Chapin (1947) para un repaso de experimentos de orientación empírica realizados en las primeras décadas del siglo $\mathrm{XX}$.

EMPIRIA. Revista de Metodología de Ciencias Sociales. N. ${ }^{\circ} 12$, julio-diciembre, 2006, pp. 89-110.

ISSN: 1139-5737 


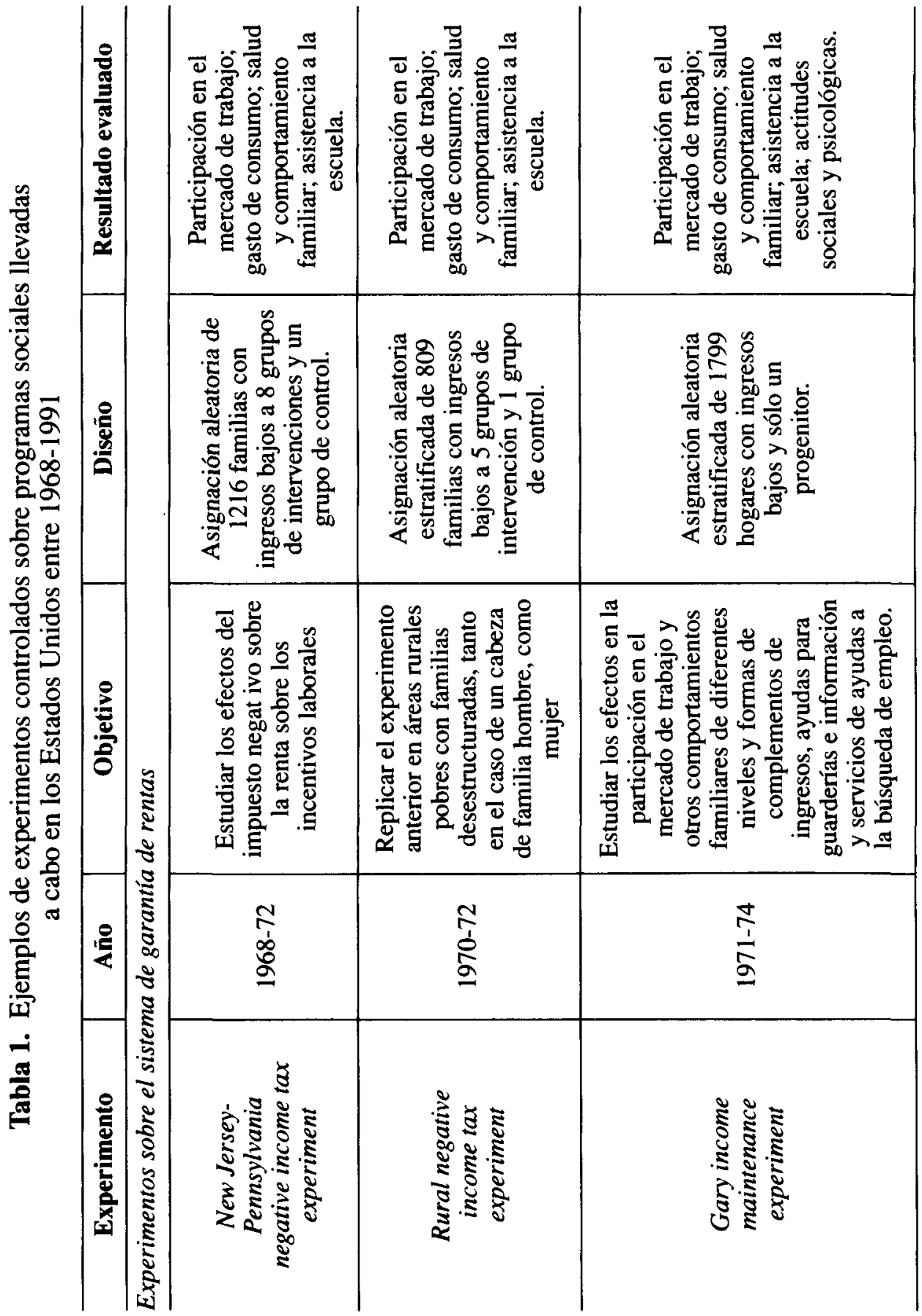




\begin{tabular}{|c|c|c|c|c|c|}
\hline 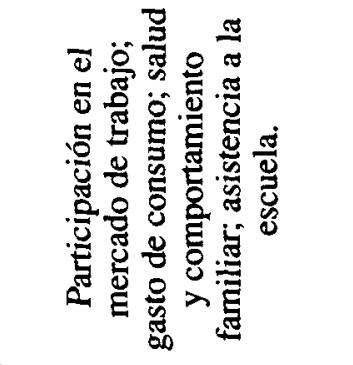 & & 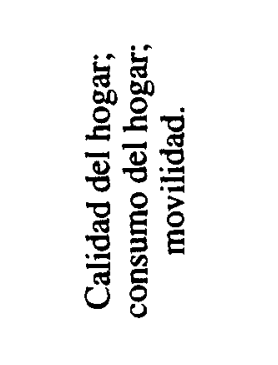 & & 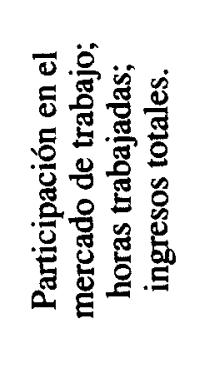 & 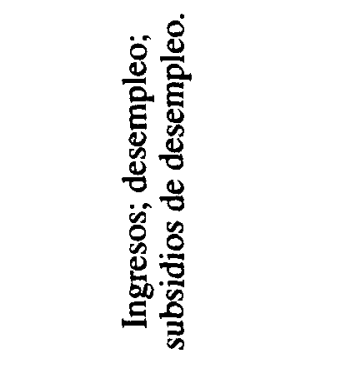 \\
\hline 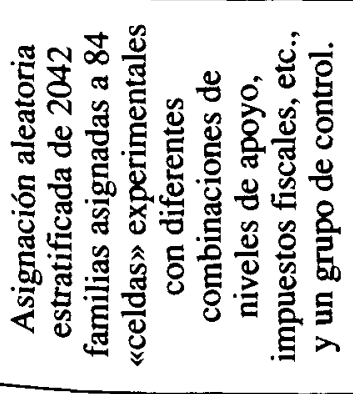 & & 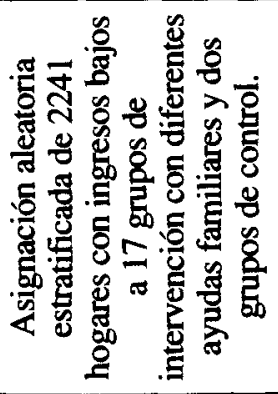 & & 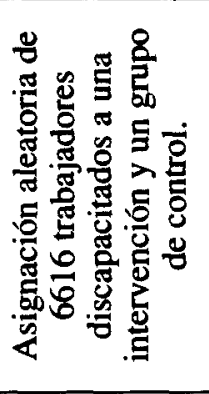 & 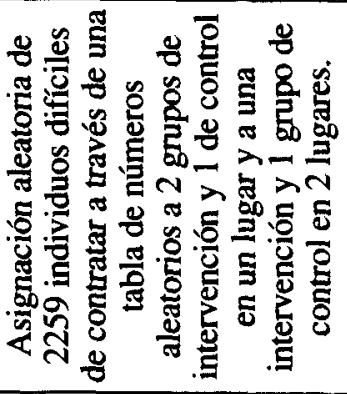 \\
\hline 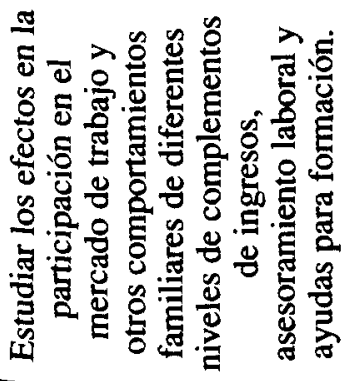 & & 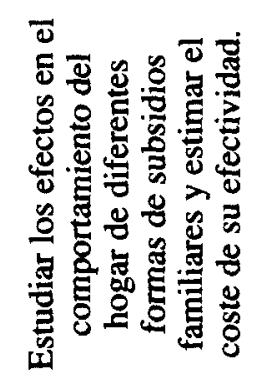 & & 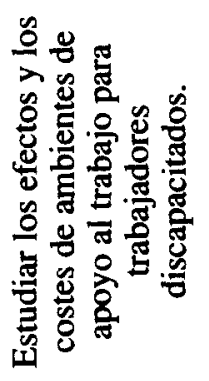 & 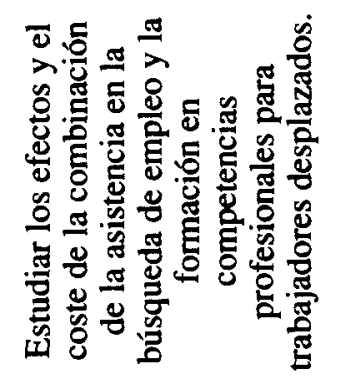 \\
\hline $\begin{array}{l}\bar{\sigma} \\
\frac{\hat{\rho}}{2}\end{array}$ & & 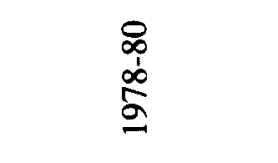 & $\frac{5}{3}$ & $\frac{\infty}{\frac{\infty}{n}}$ & 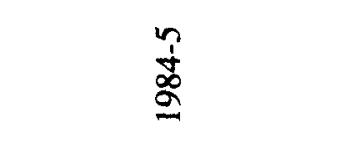 \\
\hline 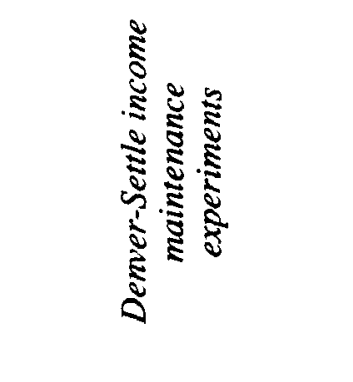 & 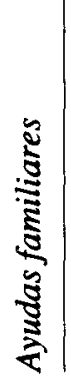 & 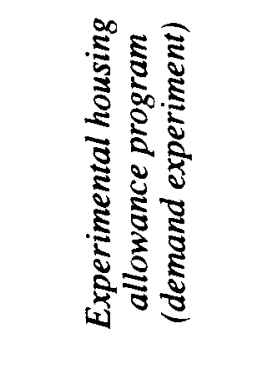 & $\begin{array}{l}\text { ปे } \\
0 \\
0 \\
\vdots \\
5 \\
5 \\
5 \\
5 \\
5 \\
5 \\
5 \\
5 \\
5\end{array}$ & 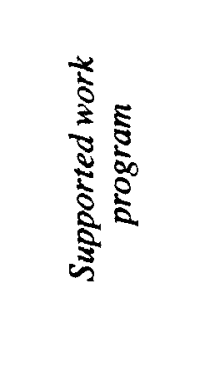 & 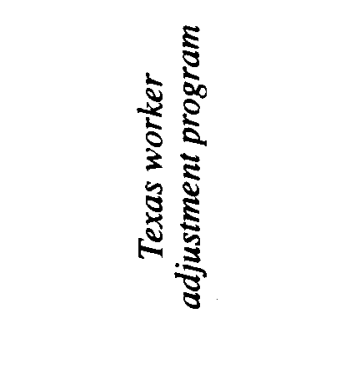 \\
\hline
\end{tabular}




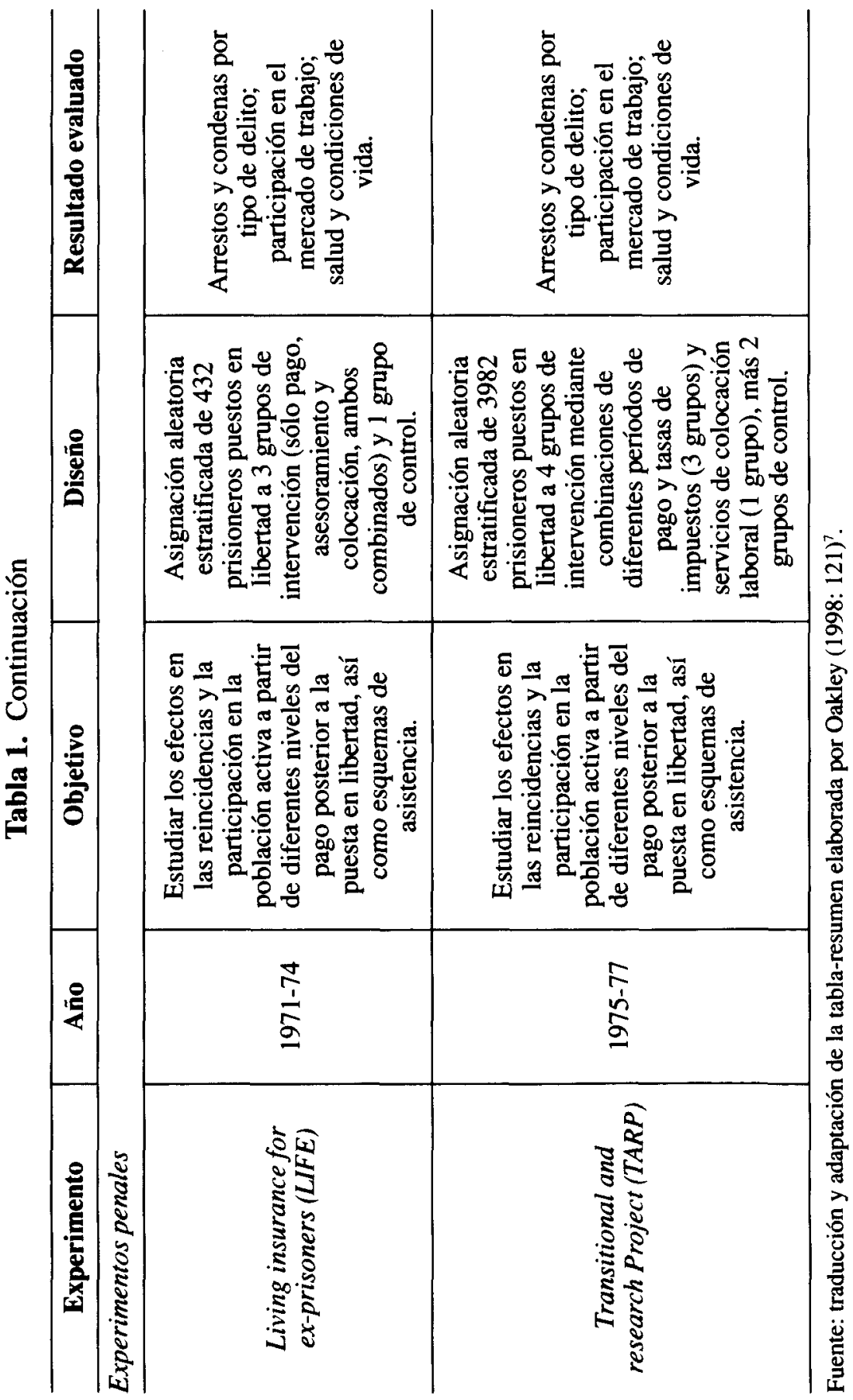


oportunidad. El objetivo era siempre determinar el efecto que cada programa de intervención tenía sobre una determinada población.

El elemento central de este tipo de experimentos es el control de las variables superfluas. En otras palabras, lo que importa es determinar, con un alto grado de confianza, el efecto producido por la introducción de una determinada variable independiente (la intervención) sobre la variable dependiente (sea ésta la participación en el mercado de trabajo, un cambio en las condiciones de vida, etc.). Dicho control es alcanzado de forma privilegiada a través de dos mecanismos, a saber: la utilización de grupos de control, que permiten aislar el efecto producido por la variable independiente introducida; y la asignación aleatoria de los sujetos experimentales a los grupos donde se produce la intervención y a los grupos de control. Este último mecanismo fue utilizado profusamente ante la imposibilidad de controlar todas las variables superfluas que podrían distorsionar la medición de los resultados de la intervención en cuestión. Desde luego, la búsqueda de un mayor control de las variables que intervienen en el experimento llevó a estos científicos sociales a emplear diseños experimentales bastante sofisticados ${ }^{8}$.

En este trabajo no nos interesan tanto los detalles de estos diseños experimentales de orientación empírica, como las diferencias de éstos con los experimentos de orientación teórica que abordaremos a continuación. De los ejemplos señalados anteriormente se deriva que los primeros pretenden responder a una cuestión eminentemente empírica, por ejemplo: ¿hasta qué punto los ex presidiarios que participan en un programa de reinserción social tienen más posibilidades de alcanzar un empleo estable fuera de la cárcel? La respuesta, positiva o negativa, a esta pregunta nos conducirá a la posibilidad de establecer una regularidad empírica del tipo: aquellos ex presidiarios que participan en un programa de reinserción social consiguen, en media, mejores empleos. Chapin (1947) hablaba de experimentos ex post facto en este caso. En resumen, se trata de experimentos que emplean muestras representativas, que investigan una posible regularidad empírica en la sociedad y cuya manipulación de la realidad (si es que ésta existe) se limita a controlar y diferenciar mediante técnicas estadísticas la población que se ha visto expuesta a algún tipo de intervención de aquella que no lo ha sido?.

${ }^{7}$ Con respecto a la tabla original presentada por Oakley, hemos respetado el inglés original de los nombres de los experimentos. Además hemos eliminado las referencias exactas de cada uno de los experimentos ya que el objetivo aquí sólo es mostrar, aprovechando el trabajo de Oakley, las características básicas del tipo de experimentos de orientación empírica a los que nos hemos referido más arriba.

${ }^{8}$ Ver, por ejemplo, el trabajo de Campbell y Stanley (1963) o el capítulo sobre diseños experimentales del libro de Moser y Kalton (1971).

${ }^{9}$ A partir de la década de los ochenta este tipo de diseño experimental de orientación empírica cay6 en desuso en los Estados Unidos. Oakley (1998: 1241) ofrece dos razones que influyeron en esta decadencia. Una primera causa fue el desencanto con la aparente ineficacia (a veces los efectos aparentemente contraproducentes) de las intervenciones, tal y como fue observado en algunas de las evaluaciones. Una segunda causa radicaba en que los políticos se mostraban a menudo impacientes con el largo período de tiempo que necesitaban las evaluaciones para aportar resultados concluyentes.

EMPIRIA. Revista de Metodología de Ciencias Sociales. N. 12, julio-diciembre, 2006, pp. 89-110. ISSN: 1139-5737 


\section{Experimentos de orientación teórica}

Los experimentos de orientación teórica, por el contrario, no analizan directamente una situación social, sino que buscan contrastar la validez de una determinada teoría, hipótesis teórica o mecanismo explicativo. Al tratarse de un método importado de la economía, en general, esta teoría suele ser la teoría de la elección racional (TER); aunque el desarrollo experimentado por este tipo de método experimental de orientación teórica hace que ya se cuestionen las propias teorías que han surgido como respuestas a las limitaciones de la TER. Entre los trabajos pioneros de esta corriente se encuentran los trabajos en el campo de la teoría de la decisión llevados a cabo a lo largo de la década de los cincuenta, cuyo principal exponente es el trabajo de Maurice Allais (1953), aunque, sin lugar a dudas, el pistoletazo de salida de la revisión experimental de los principales supuestos de la TER fue dado por la publicación del trabajo Kahneman y Tversky $(1979)^{10}$. Pero antes de detenernos en las características fundamentales de este tipo de diseños experimentales, volvamos a la relación entre realidad, teoría y experimento a la que nos referíamos con anterioridad. Kanazawa (1999) insiste en la orientación teórica de los experimentos de laboratorio - los experimentos puros de los que hablaba Greenwood-del siguiente modo:

uno nunca generaliza los hallazgos empíricos de los experimentos de laboratorio a una población mayor en la que está interesado. Como han argumentado muchos en el pasado [...], el propósito de llevar a cabo experimentos de laboratorio en cambio es someter a prueba una teoría abstracta y general cuyas condiciones de posibilidad están enunciadas claramente, y, una vez contrastada ésta, es la teoría, no los datos experimentales, la que es generalizada a situaciones naturales de interés que se ajustan a sus condiciones de posibilidad (p. 452).

Más adelante afirma:

un experimento de laboratorio cuidadosamente diseñado para ajustarse a todas las condiciones de posibilidad de la teoría no es más que un caso empírico de los que la teoría explica. Sin embargo, tampoco es menos.

Por tanto, al contrario que los experimentos de orientación empírica, en los que un caso empírico real era analizado, los experimentos de orientación teórica se proponen reconstruir en el laboratorio una determinada teoría y, de este modo, estudiar el efecto producido por la manipulación explícita de los distintos componentes de la misma. La Figura 1 nos puede ayudar a visualizar los argumentos introducidos por Kanazawa.

${ }_{10}$ Para un repaso de algunos de los experimentos simples que han contribuido a poner en duda la validez descriptiva de la TER, incluyendo el propio trabajo de Allais, ver Aguiar (2004). 


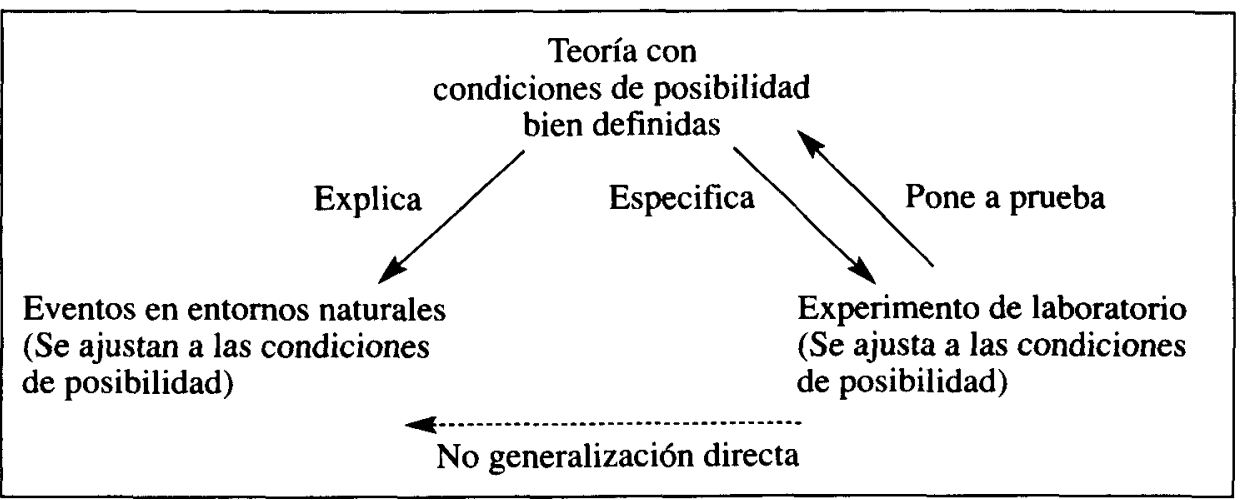

Fuente: traducido de Kanazawa (1999a: 452).

Figura 1. Diseño apropiado del experimento.

El primer paso para la realización de un experimento de laboratorio (de orientación teórica) es disponer de una teoría cuyo alcance esté bien definido. Una vez que disponemos de ésta, nuestro objetivo será intentar que la especificación que se realiza de la misma en el experimento se ajuste en la mayor medida posible al alcance y condiciones de posibilidad de dicha teoría. Este ajuste es lo que se denomina validez interna del experimento, que se preocupa de «la cuestión de si mediante el experimento se obtiene una medida verdadera del efecto de la variable independiente [en la variable dependiente]" (Moser y Kalton: 1971: 220). Si se diseña un experimento para poner a prueba una teoría preexistente, la validez interna de los resultados obtenidos, ya vayan éstos en la dirección de la confirmación (parcial) o refutación (parcial) de la teoría, dependerá de lo capaces que seamos para recrear los distintos aspectos de la teoría en la situación experimental concreta. Este argumento quizá pueda quedar más claro con un ejemplo concreto.

Pensemos en la conocida proposición teórica de Mancur Olson con la que este economista norteamericano predecía la imposibilidad de la acción colectiva en grupos lo suficientemente grandes y en ausencia de incentivos selectivos de carácter individual. En una afirmación que ya se ha hecho célebre, Olson afirmaba que «los individuos racionales y auto-interesados no actuarán para alcanzar sus intereses comunes» (Olson, 1965: 2). Algunos años más tarde, la estructura estratégica de la logica de la acción colectiva fue representada formalmente a través del juego del dilema del prisionero de $n$ personas (Hardin, $1971 ; 1982)$. Una generalización del juego del dilema del prisionero a $n$ personas nos conduce a un juego de bien público (Kollock, 1998). Tanto el juego del dilema del prisionero como el juego de bien público han sido las dos situaciones estratégicas que han recibido una mayor atención por parte de la economía experimental. En la actualidad podemos hablar de miles de experimentos en los que ambas estructuras estratégicas han sido estudiadas, haciendo cada uno de 
ellos hincapié en aspectos diferentes de ambas ${ }^{11} \cdot ¿$ Quiere esto decir que después de tal cantidad de experimentos sabemos más acerca del problema que planteaba Olson? La respuesta correcta a esta pregunta es que actualmente sabemos mucho más acerca de cómo las personas se comportan ante un dilema del prisionero o un juego de bien público y esto, a su vez, nos permite discutir los fundamentos teóricos que llevaron a Olson a desarrollar su hipótesis teórica básica. Pero nunca podríamos decir que la obtención de evidencia experimental sobre la cooperación de los individuos en este tipo de situaciones experimentales refrenda o invalida la propuesta olsoniana. Así, el experimentador será el responsable de que la situación experimental se ajuste a las características exactas del dilema del prisionero, el teórico de juegos deberá rendir cuentas de que dicho juego capta la esencia del problema de la ausencia de cooperación en el comportamiento colectivo y el teórico social, finalmente, será el encargado de defender que la proposición teórica que afirma que los individuos tienen una estrategia dominante no cooperativa bajo las circunstancias descritas por Olson y Hardin goza de suficiente soporte empírico ${ }^{12}$.

Sugden (2005) ha resumido esta triangulación entre el experimento, la teoría y el mundo real del siguiente modo:

un experimento es una comprobación de una teoría preexistente. Dicha teoría pretende ser una representación de ciertas características del mundo. Al poner a prueba la teoría, el experimentador construye una situación de laboratorio que en algunos aspectos se parece a la teoría (y así representa el mundo en tanto que la teoría lo hace), mientras que en otros aspectos representa el mundo de forma diferente, al reemplazar ciertos presupuestos de la teoría por el comportamiento de sujetos humanos autónomos (p. 180).

Por tanto, aunque «la investigación experimental ha descubierto regularidades empíricas para las que, por el momento, no existen explicaciones teóricas bien desarrolladas» (Sugden, 2005: 182), éste no es su objetivo fundamental, sino el de poner a prueba teorías preexistentes. Terminaremos este apartado con otra cita de Kanazawa (1999a) que deja meridianamente claro el argumento que estamos desarrollando:

Los experimentos de laboratorio no son métodos de descubrimiento. Sin una teoría general y abstracta con condiciones de posibilidad clara-

1 Para un repaso de los diversos tipos de experimentos diseñados para estudiar estos juegos ver Ledyard (1995). Para un resumen más recientes de las aportaciones de la economía experimental al estudio de la cooperación humana, ver Ostrom (2000).

${ }^{12}$ El problema de la cooperación en la acción colectiva es uno de los temas estrella en los experimentos sociologicos de orientación teórica. Otros temas sobre los que se han realizado un número considerable de experimentos teóricos en los últimos años son: «análisis de organizaciones» (Kanazawa, 1999a, 1999b), «confianza» (Kollock, 1994; Hayashi y Yosano, 2005; Yamagishi et al., 2005), «redes sociales» (Smatka et al., 1999; Willer, Troyer y Lovaglia, 2005), «creencias sociales» (Markovsky y Thye, 2001), «diferencias de género» (Shelly y Munroe, 1999) o «convenciones sociales» (Büchner, Güth y Miller, 2005; Miller, 2006). 


\begin{abstract}
mente establecidas, éstos no pueden decimos nada más allá de cómo algunos "estudiantes universitarios» se comportan en un entorno dado altamente artificial; no tienen ninguna utilidad. Sin embargo, con una teoría, los experimentos son inigualables como un medio de evaluar y mejorar la teoría (p. 455).
\end{abstract}

Aunque se puede deducir de lo comentado hasta el momento, en el siguiente apartado nos ocuparemos de uno de los aspectos más polémicos, aunque también uno de los que provocan más equívocos, de los experimentos de laboratorio: su validez externa.

\title{
3. LA VALIDEZ EXTERNA DE LOS EXPERIMENTOS DE ORIENTACIÓN TEÓRICA
}

La validez externa de una investigación tiene que ver con la posibilidad de generalizar los resultados obtenidos en la misma a una población más amplia, otros entornos de investigación, otras variables de tratamiento $\mathbf{u}$ otras variables de medición (ver, entre otros, Moser y Kalton, 1971: 220; Campbell y Stanley, 1963: 5). Intuitivamente se trata de una problemática que asociamos a las encuestas, sondeos de opinión y otros tipos de técnicas de investigación que pretenden, a partir de una determinada muestra, determinar las opiniones, actitudes y comportamientos de una población mayor. Sin embargo, este tipo de requisito técnico no solamente ha preocupado a los profesionales de las encuestas, sino, como veíamos anteriormente, también a otro tipo de investigadores sociales como aquéllos que han empleado diseños experimentales. Ahora bien, y esta es una de las tesis centrales de este artículo, la preocupación por la validez externa no tiene sentido cuando nos referimos a experimentos de orientación teórica. La siguiente afirmación de Kanazawa (1999a) no puede ser más clara al respecto:

Una de las críticas más comunes a los experimentos de laboratorio es la ausencia de validez externa. Los críticos a menudo acusan a los experimentos de laboratorio de tener una validez externa débil. Esto es falso; los experimentos de laboratorio no tienen validez externa alguna (p. 452).

Esta afirmación puede rechinar en los oídos del sociólogo contemporáneo, tan preocupado por determinar hasta qué punto sus resultados son generalizables, pero recordemos que los experimentos de orientación teórica no suponen un método de descubrimiento, sino un método mediante el cual poner a prueba determinadas teorías. Volvamos por un momento al ejemplo del problema de la cooperación en la acción colectiva introducido en el apartado anterior. Según numerosos estudios experimentales (ver Ostrom, 2000: 140), aquellos sujetos que creen que otros van a cooperar en una situación de acción colectiva determinada tienen una mayor probabilidad de cooperar en la misma. En otras pala- 
bras, la expectativa de un comportamiento cooperativo del resto supone una fuerza motivadora del comportamiento cooperativo propio, lo que nos puede llevar a concluir que se está activando un mecanismo de «cooperación condicional» $o$, en términos generales, algún tipo de comportamiento recíproco. Este resultado experimental, habiendo sido replicado en innumerables ocasiones, se ha convertido en uno de los resultados más robustos que ha aportado la economía experimental al estudio del problema de la cooperación humana. Sin embargo, este resultado no nos dice nada acerca de si es más probable observar pautas de reciprocidad en España o en Alemania, entre jovenes o entre personas mayores, entre votantes de izquierda o votantes de derecha. Para abordar estas últimas cuestiones sí que sería necesario plantear el problema de la validez externa. Lo que en realidad aporta el mecanismo descrito anteriormente es que, cuando nos dispongamos a construir nuestros modelos teóricos y modelos matemáticos, tendremos que tener en cuenta que las «expectativas de reciprocidad" pueden tener cabida en la explicación de por qué un determinado grupo de personas podría cooperar para la obtención de un bien colectivo ${ }^{13}$.

A pesar de lo expuesto anteriormente, la cuestión de la validez externa sigue siendo una cuestión disputada en la economía experimental. Entre otros motivos porque esta subdisciplina en el campo de la economía ha descubierto regularidades empíricas para las que, por el momento, no existen explicaciones teóricas bien elaboradas (Sugden, 2005: 182). Siendo esto así, el problema que se plantea es si estas regularidades empíricas, extraídas del estudio sistemático de situaciones de laboratorio, nos dicen algo acerca de cómo funcionan realmente las instituciones económicas y sociales. Para responder a esta cuestión plantearemos la pregunta acerca de la validez externa de la investigación de forma inversa a como normalmente es planteada. No nos preguntaremos acerca de hasta qué punto los datos derivados de la investigación experimental responden a las pautas generales observadas en el mundo real, sino que nos preguntaremos por los posibles factores que podrían hacer que ambos ámbitos fueran diferentes de un modo sustancial.

Las diversas reflexiones metodológicas a propósito de la especificidad de los experimentos de laboratorio se han centrado en el problema de la artificialidad. Ésta, siendo una característica definitoria de los experimentos de laboratorio - el propio investigador ha creado ex ante la situación que pretende estudiarplantea algunos interrogantes metodológicos que apuntan directamente a la validez externa de la investigación. Por ejemplo, en determinados casos, «la abstracción de algunos diseños experimentales puede provocar que algunas de las

${ }_{13}$ Alguien podría afirmar que los trabajos de Axelrod $(1981 ; 1984)$ sobre la cooperación en juegos repetidos ya habían adelantado tal mecanismo teórico. En este caso, la experimentación puede servir para estudiar con «sujetos reales» cómo funciona en la práctica dicho mecanismo, permitiendo plantear preguntas del tipo: $i$ tiene el conocimiento del horizonte temporal una influencia sobre las expectativas de reciprocidad?, ¿influye el tamaño del grupo en el comportamiento recíproco?, ¿supone la reciprocidad una preferencia intrínseca o un comportamiento estratégico por parte de egoístas maximizadores?

EMPIRIA. Revista de Metodología de Ciencias Sociales. N. ${ }^{\circ}$ 12, julio-diciembre, 2006, pp. 89-110. 
variables que afectan de forma importante las decisiones en el mundo real queden fuera del diseño del laboratorio y por ello no se puedan obtener conclusiones aplicables al exterior» (Rey, 2006: 8). Por su parte Sugden (2005: 180) ha señalado que en multitud de ocasiones se ha criticado a los diseños experimentales por la ausencia de determinadas características supuestamente necesarias en toda investigación. Entre estas características destacan las siguientes:

- los sujetos no son representativos de la población de agentes cuyo comportamiento económico la teoría está diseñada para explicar;

- éstos no tienen la oportunidad adecuada de aprender por ensayo y error cómo actuar racionalmente, del mismo modo que los agentes económicos lo hacen en situaciones reales;

- los incentivos que han de actuar de forma óptima son menores en el laboratorio que fuera del mismo;

- la naturaleza abstracta de las tareas experimentales elimina pistas que, en el campo, ayudarían a las personas a orientarse en problemas de decisión;

- las normas sociales que guían la interacción en el campo no son reproducidas adecuadamente en el laboratorio.

Tratar cada uno de estos retos metodológicos supone responder a la pregunta previa acerca de la relación entre la situación de laboratorio y el mundo real. Si esta relación es directa, sin la mediación de ninguna teoría, todos los problemas señalados anteriormente cobran una especial relevancia. Por el contrario, si el experimento está construido a partir de una teoría sólida, con unas condiciones de posibilidad bien definidas, un buen diseño interno de la investigación puede conducirnos a no necesitar plantearnos el problema de la validez externa, como ya señalaba Kanazawa en el extracto introducido más arriba.

Una vez que hemos discutido las dos cuestiones metodológicas planteadas en la introducción -qué entendemos por método experimental y cuáles son sus fuentes de validez - vamos a concluir este trabajo haciendo referencia a dos cuestiones clave, y a la vez controvertidas, del tipo de experimentos predominantes en la economía experimental ${ }^{14}$.

\section{LA ESPECIFICIDAD DE LOS EXPERIMENTOS ECONÓMICOS}

Como hemos señalado con anterioridad, la reciente utilización de experimentos de orientación teórica por parte de determinados sociólogos ha tenido

14 Aquí se establece una correspondencia entre lo que denominamos experimentos de orientación teórica y el tipo de experimento predominante en la economía experimental contemporánea. En realidad no todas las investigaciones que emplean el método experimental en econọmía persiguen un objetivo puramente teorico, aunque éste sí es predominante. No es éste el lugar para profundizar en la heterogeneidad de los objetivos, diseños y procedimientos propios de la economía experimental. 
que ver con la incorporación al arsenal metodológico del sociólogo del tipo de diseño experimental predominante en la economía experimental contemporánea ${ }^{15}$. Ello implica dos elecciones metodológicas que no tienen por qué haber estado presentes en otros diseños experimentales en el seno de disciplinas como la psicología social o la propia sociología. En primer lugar, todas las decisiones que los agentes toman a lo largo de una sesión experimental tienen que estar relacionadas con el sistema de incentivos materiales empleado en el experimento. En segundo lugar, toda la información ofrecida a los participantes tiene que ser pública y verdadera. Dado que se trata de dos criterios que pueden resultar hasta cierto punto arbitrarios, en este apartado nos detendremos a argumentar brevemente el porqué de su uso.

\section{Uso de incentivos monetarios}

En un reciente tratado sobre metodología de la economía experimental, Francesco Guala (2005: 25) afirma que «El hábito de compensar [económicamente] a los sujetos distingue a la economía experimental de otras disciplinas vecinas, como la psicología experimental.» Aunque este requisito de pagar a los participantes por las tareas realizadas en el experimento pueda resultar innecesario para otras tradiciones experimentales, no debe sorprender que el método experimental aplicado a una ciencia - la economía - que pone el énfasis en los incentivos que tienen los agentes para tomar decisiones, derive en una preocupación importante por el mejor modo de recrear dichos incentivos en el laboratorio. Dicho esto, ¿qué requisitos deben cumplir los sistemas de incentivos empleados en los diseños experimentales?

Rey (2006: 3) señala que «para que los incentivos del experimento permitan cierto control sobre las preferencias de los sujetos es necesario que se cumplan tres condiciones:

1. Contingencia: La cantidad de incentivo recibido debe depender al menos de la decisión tomada por el propio sujeto.

2. Dominancia: Los cambios en la satisfacción del sujeto con el experimento se tienen que deber fundamentalmente a cambios en las cantidades de dinero recibido.

3. Monotonicidad: Un mayor pago del incentivo debe ser siempre preferido a un pago menor, y los sujetos no deben llegar a estar saciados».

Cuando se cumplen estas tres condiciones el experimentador puede asumir que los comportamientos observados a lo largo del experimento dependen directamente de los incentivos económicos empleados en el mismo. Pero esto, por

15 Para un panorama bastante completo de los logros y las limitaciones de la economía experimental, ver la compilación de Kagel y Roth (1995) y el texto de Camerer (2003).

EMPIRIA. Revista de Metodología de Ciencias Sociales. N. ${ }^{\circ}$ 12, julio-diciembre, 2006, pp. 89-110. 
supuesto, no quiere decir que todo comportamiento que no se ajuste a la maximización de los incentivos materiales propuestos en el experimento deba ser rechazado. Muy al contrario, el uso explícito de incentivos materiales ha permitido el estudio controlado de motivaciones no materiales, tales como el altruismo, la generosidad o el comportamiento guiado por normas morales y convenciones sociales $^{16}$. Nos detendremos en un tipo de experimento bastante popular en la economía experimental para ilustrar este argumento: el juego del dictador.

El juego del dictador (dictator game) es uno de los juegos más simples empleados por los economistas experimentales para el estudio de las motivaciones humanas. En éste participan dos jugadores, el «proponente» (proposer) y el «receptor» (receiver). El proponente determina una asignación (división) de alguna dotación (por ejemplo, una cantidad económica obtenida en un premio). El receptor en este caso simplemente recibe el remanente de la dotación no asignada a sí mismo por el proponente. El receptor desempeña un papel completamente pasivo: no tiene ninguna influencia estratégica sobre el resultado del juego ${ }^{17}$. Este juego ha sido utilizado para poner a prueba el modelo del homo oeconomicus; así, si los individuos estuvieran preocupados sólo por su propio bienestar, los proponentes se asignarían a sí mismos la totalidad de la «tarta» que se ha de repartir y no le darían nada a los receptores. Sin embargo, numerosos estudios, incluyendo un amplio estudio comparativo trans-cultural (ver Henrich et al., 2004), han demostrado que los proponentes asignan cantidades de la dotación por encima de la predicción teórica («dar cero»). Estos resultados pueden hacernos conjeturar dos tipos de explicaciones: bien los proponentes no son capaces de maximizar su utilidad esperada o bien la función de utilidad de los proponentes incluye los beneficios obtenidos por los otros. Ambas explicaciones, como mínimo, plantean retos serios para la teoría de la elección racional más estándar.

El ejemplo del juego del dictador nos ha servido para tener una primera intuición acerca de cómo son utilizados los incentivos monetarios en la economía experimental, así como acerca de las interpretaciones que podemos hacer a propósito de las motivaciones individuales a partir de los comportamientos observados en el experimento. En otros casos, los incentivos permiten reconstruir los incentivos que delimitan el sistema de interacción contenido en una matriz de juegos concreta.

${ }^{16}$ Mecanismos experimentales como el denominado «juego del ultimátum» (Güth, Schmittberger y Schwarse, 1982; Güth y Tietz, 1990) o el «juego del dictador» (Kahneman, Knetsch y Thaler, 1986) han contribuido a impulsar el estudio y el análisis de motivaciones no materiales presentes en la toma de decisiones económicas y sociales. A su vez este tipo de estudios experimentales han contribuido de forma muy importante al desarrollo de la denominada «economía del comportamiento", una rama de la teoría económica en la que se ponen sistemáticamente a pruebas los principales fundamentos teóricos de la aplicación de la teoría de la elección racional al campo económico. Para un exhaustivo trabajo que vincula los resultados experimentales con el avance de la «economía del comportamiento», ver Camerer (2003).

$17 \mathrm{Al}$ no existir interacción estratégica, numerosos autores niegan que se pueda llamar juego a este tipo de diseño. En aras de la claridad expositiva aquí seguiremos hablando del «juego del dictador». 


\section{Prohibición del engaño (deception)}

De acuerdo con las convenciones imperantes en la economía experimental, toda la información que se suministre a los sujetos experimentales debe ser verdadera y el experimento debe ajustarse completamente a ésta. Como señala Rey (2006: n. 15), «la preocupación por este tema es tal que existe un acuerdo tácito por el que no se acepta la publicación de ningún artículo en el que se sospeche que ha habido 'engaño'» ${ }^{18}$. La prohibición del engaño en economía experimental incluye: el engaño acerca del propósito del experimento, acerca de los pagos que el sujeto puede obtener o acerca de la caracterización de los sujetos con los que el participante está interactuando (Croson, 2002: 940).

Se han señalado dos razones en defensa de esta prohibición (Croson, 2002). En primer lugar, la validez de los experimentos económicos está basada fundamentalmente en la conexión entre el comportamiento de los sujetos experimentales y el pago esperado. Si el sujeto experimental pone en duda dicha conexión el propio experimento pierde todo su sentido. En segundo lugar, en economía experimental, la confianza que los participantes depositan en el experimentador es considerada un bien público. Por ejemplo, si un sujeto participa en repetidas ocasiones en un experimento e independientemente de su implicación en el mismo siempre recibe una suma constante de dinero, puede tender a desconfiar en la palabra del experimentador y, de este modo, comportarse de una manera que resulte completamente impredecible al no depender de los incentivos propuestos.

Al igual que la cuestión del empleo de incentivos monetarios, la prohibición de utilizar el engaño para estudiar ciertas pautas de comportamiento humano, aun siendo una cuestión susceptible de debate, no está sujeta a discusión en el estado actual de la economía experimental y es aceptada como un precepto que hay que cumplir. En la actualidad existen numerosos debates metodológicos abiertos acerca de éstas y otras cuestiones particulares de la economía experimental; prueba de ello son trabajos como el de Francesco Guala (2005) o el monográfico de la Journal of Economic Methodology coordinado por Robert Sugden en 2005. El presente trabajo sólo pretende incitar al sociólogo a tomar parte en un debate metodológico en el que sin duda tiene mucho que decir.

\section{CONCLUSIONES}

Este artículo ha pretendido ser una introducción al método experimental, en general, y su vertiente de orientación teórica, en particular. Para ello hemos planteado primero la cuestión de qué entendemos por método experimental - un

18 En este sentido, la economía experimental se sitúa bastante lejos de las posiciones mantenidas por los psicólogos sociales. Recordemos sin ir más lejos los famosos experimentos llevados a cabo por Stanley Milgram sobre el acatamiento a la autoridad. En éstos se hacía creer a los sujetos experimentales que estaban infringiendo un castigo físico real a otros participantes, siendo éste sólo el más llamativo de los engaños a los que estaban sometidos los mismos. 
método que pretende establecer relaciones causales a partir del estudio sistemático y controlado de diferentes situaciones de contraste-, así como qué tipos ideales de diseños podemos encontrar, en concreto, hemos distinguido entre experimentos de orientación empírica y experimentos de orientación teórica. A continuación, hemos planteado los problemas de validez a los que este último tipo de experimentos tiene que hacer frente.

La cuestión determinante a la hora de evaluar un experimento de orientación teórica es su validez interna, es decir, hasta qué punto nuestro diseño mide lo que, en teoría, pretendemos medir. La validez interna de este tipo de experimentos depende críticamente de una adecuada triangulación de tres elementos: mundo real, teoría y diseño experimental. Para obtener una validez interna alta debemos disponer de una teoría que explique adecuadamente la realidad y que, además, esté lo suficientemente bien definida como para que pueda ser reproducida en el laboratorio de forma satisfactoria.

Si la validez interna es lo que nos permite discriminar entre buenos y malos experimentos de orientación teórica, otro tipo de fuente de validez, la validez externa, desempeña un papel secundario. Si nuestro objetivo es analizar el comportamiento de una teoría dentro de su alcance y sus condiciones de posibilidad, la pregunta a propósito de la generalización de los resultados de un experimento concreto no tiene cabida. En todo caso, deberíamos plantearnos en qué medida la situación recreada en el laboratorio es sustancialmente distinta al tipo de mecanismo social observado en la realidad que pretendemos estudiar. Varios autores coinciden en resaltar el problema de la artificialidad propia de las situaciones de laboratorio como una de las amenazas más importantes para la validez externa de los experimentos. Sin embargo, en el caso de los experimentos de orientación teórica, esto nos conduce de nuevo al problema de la especificación del experimento, es decir, su validez interna; volviendo a constatar la intrascendencia de la preocupación por la validez externa cuando nos referimos a este tipo de experimento.

Por último, nos hemos detenido en dos características claves de los diseños experimentales en el campo de la economía experimental: el uso de incentivos monetarios y la prohibición del engaño. Dado que este tipo de experimentos ocupan un lugar cada vez más central en el seno de la sociología, hemos creído conveniente aportar algunas aclaraciones metodológicas con relación a su empleo.

En definitiva, este artículo sólo ha pretendido dar algunas pinceladas metodológicas sobre un tipo de diseño de investigación que, tras haber revolucionado el campo de la teoría económica, empieza a prodigarse en investigaciones sociológicas. Dado que esto es así, lo más adecuado es tener siempre presentes las oportunidades y los riesgos que el mismo entraña.

\section{BIBLIOGRAFÍA}

Aguiar, F. (2004), «Teoría de la decisión e incertidumbre: modelos normativos y descriptivos», Empiria, n. ${ }^{\circ}$, pp. 139-60.

EMPIRIA. Revista de Metodología de Ciencias Sociales. N. ${ }^{\circ} 12$, julio-diciembre, 2006, pp. 89-110. 
Allais, M. (1953), « Le Comportement De L'homme Rationnel Devant Le Risque: Critique Des Postulats Et Axiomes De L'ecole Americaine » Econometrica, vol. 21: pp. 503-46.

AXELROD, R. (1981), «The Emergence of Cooperation among Egoists», American Political Science Review, vol. 75: pp. 306-18.

- (1986), La Evolución De La Cooperación, Madrid, Alianza Editorial.

Brown, S. R. y L. E. Melamed (1990), Experimental Design and Analysis, Thousand Oaks, Sage.

BüCHNER, S., W. GüTh y L. M. MiLler (2005), «Conventions for Implementing Conventions: An Evolutionary and Experimental Analysis», Discussion Papers on Strategic Interaction, $\mathbf{n}^{\circ}$ 21/05, Max Planck Institute of Economics, Jena, Alemania.

CAmerer, C. F. (2003), Behavioral Game Theory, Princeton, Princeton University Press.

CAMPBell, D. T. y J. C. STANLEy (1963), Experimental and Quasi-Experimental Designs for Research, Boston: Houghton Mifflin Company.

Chapin, F. S. (1931), Experimental Designs in Sociological Research, Nueva York, Harper y Row.

Croson, R. (2002), «Why and How to Experiment: Methodologies from Experimental Economics», University of Illinois Law Review, 2002: pp. 921-45.

GreENwoOd, E. (1976 [1945]), Experimental Sociology. A Study in Method, Nueva York, Octagon Books.

GÜTH, W., R. SCHMITTBERGER y B. SCHWARZE (1982), «An Experimental Analysis of Ultimatum Bargaining», Journal of Economic Behavior \& Organization, vol. 3 (4): pp. 367-88.

GütH, W. y R. TIETz (1990), «Ultimatum Bargaining Behavior - a Survey and Comparison of Experimental Results", Journal of Economic Psychology, vol. 11 (3): pp. 417-49.

HaRdin, R. (1971), «Collective Action as an Agreeable N-Prisoners` Dilemma» Behavioral Science, vol. $16(5)$ : pp. 472-81.

- (1982), Collective Action, Londres, John Hopkins University Press.

HAYASHI, N. y A. YOSANO (2005), "Trust and belief about others: Focusing on judgment accuracy of others' trustworthiness», Sociological Theory and Methods, Vol. 20 (1), pp. 59-80.

Henrich, J., R. Boyd, S. Bowles, C. Camerer, H. Gintis, R. Mcelreath y E. Fehr (2001), "In search of Homo economicus: Experiments in 15 Small-Scale Societies", American Economic Review, vol. 91(2), pp. 73-79,

Kahneman, D., J. Netsch y R. ThaleR (1986), «Fairness and the Assumptions of Economics», Journal of Business, vol. 59: pp. 285-300.

KAHNEMAN, D. y A. TVERSKy (1979), «Prospect Theory: An Analysis of Desicion under Risk», Econometrica, vol. 47, pp. 263-91.

Kanazawa, S. (1999a), «Using Laboratory Experiments to Test Theories of Corporate Behavior», Rationality and Society, vol. 11 (4), pp. 443-61.

- (1999b), «Testing Macro Organizational Theories in Laboratory Experiments», Social Sciences Research, vol. 28, pp. 66-87.

KoLLOCK, P. (1994), «The Emergence of Exchange Structures: An Experimental Study of Uncertainty, Commitment and Trust», American Journal of Sociology, vol. 100 (2), pp. 313-345.

- (1998), «Social Dilemmas: The Anatomy of Cooperation», Annual Review of Sociology, vol. 24, pp. 183-214. 
LEDYARD, J. (1995), «Public Goods: A Survey of Experimental Research», en J. Kagel y A. Roth (eds.), Handbook of Experimental Economics, Princeton, Princeton University Press.

LEVIN, I. P. (1999), Relating Statistics and Experimental Design. An Introduction, Thousand Oaks, Sage.

MARKovSKy, B y S. R. Thye (2001), «Social influence on paranormal beliefs», Sociological Perspectives, vol. 44 (1), pp. 21-44.

MiLL, J. S. (1891), System of Logic Ratiocinative and Inductive, Londres, Longmans, Green and Co.

Miller, L. M. (2006), «The Double Nature of Conventions: An Experimental Analysis», Discussion Papers on Strategic Interaction, n. $7 / 06$, Max Planck Institute of Economics, Jena, Alemania.

Moser, C. A. y G. Kalton (1992 [1971]), «Experiments and Investigations», en C. A. Moser y G. Kalton, Survey Methods in Social Investigation, Hants: Gower, pp. 211 237.

OAKLEY, A. (1998), «Experimentation and Social Interventions: A Forgotten but Important History», BMJ 317, pp. 1239-42.

Olson, M. (1971), The Logic of Collective Action. Publics Goods and the Theory of the Group, Cambridge (MA), Harvard University Press.

Ostrom, E. (2000), «Collective Action and the Evolution of Social Norms», Journal of Economic Perspectives, vol. 14 (3), pp. 137-58.

REY, P. (2006), «Economía experimental y teoría de juegos», Mimeo.

SHELly, R. K. y P. T. MunRoE (1999), «Do women engage in less task behavior than men?», Sociological Perspectives, vol. 42 (1), pp. 49-67.

Smatka, J., J. Skvorets, T. Sozanski y J. MazuR, «Conflict in Networks», Sociological Perspectives, vol. 41 (1), pp. 49-66.

Sugden, R. (2005), «Experiment, Theory, World: A Sysposium on the Role of Experiments in Economics", Journal of Economic Methodology, vol. 12 (2), pp. 177-184.

WiLleR, R., L. TROYER y M. J. LOVAGLIA (2005), «Influence over observers of structural power: An experimental investigation», Sociological Quarterly, vol. 46 (2), pp. 263277.

Yamagishi, T., S. Kanazawa, R. Mashima y S. TeRai (2005), «Separating Trust from Cooperation in a Dynamic Relationship. Prisoners' Dilemma with Variable Dependence», Rationality and Society, Vol. 17 (3), pp. 275-308. 


\title{
RESUMEN
}

Este artículo pretende reflexionar sobre la relevancia del uso actual de un tipo particular de diseño experimental en Sociología: los experimentos de orientación teórica. Para ello, en primer lugar, repasa las características básicas del método experimental. En segundo lugar, plantea la distinción entre experimentos sociológicos de orientación empírica y experimentos sociológicos de orientación teórica. La principal diferencia entre ambos diseños radica en que el primero pretende el estudio experimental de una situación empírica concreta, mientras que el segundo tiene como objetivo poner a prueba una teoría, hipótesis teórica o mecanismo explicativo preexistentes. En tercer lugar, el artículo rechaza la necesidad de tomar en consideración el problema de la validez externa cuando nos referimos a los experimentos de orientación teórica. Por último, y debido a la influencia determinante de la economía experimental en los experimentos sociológicos de orientación teórica, se introducen dos aspectos metodológicos cruciales de los experimentos económicos: el uso de incentivos económicos y la prohibición del engaño (deception).

\section{PALABRAS CLAVE}

Método experimental, experimentos de orientación teórica, validez externa, economía experimental.

\begin{abstract}
This article aims to consider the relevance of the present use of a particular experimental design in Sociology: theory-driven experiments. To this end, firstly, the basic characteristics of the experimental method are reviewed. Secondly, the distinction between empirical experiments and theory-driven experiments is posed. The main difference between both designs is that the first one aims to the experimental study of a certain empirical situation, whereas the aim of the second one is testing pre-existing theories, theoretical hypotheses or explicative mechanisms. Thirdly, when considering theory-driven experiments, the concern for external validity is rejected. Finally, and due to the crucial influence of experimental economics in sociological theory-driving experiments, two main methodological aspects of economic experiments are introduced: the use of monetary incentives and the problem of deception.
\end{abstract}

\section{KEYWORDS}

Experimental method, theory-driven experiments, external validity, experimental economics. 